Thorax (1974), 29, 145.

\title{
A patient with respiratory obstruction in glandular fever
}

\author{
A. D. SIMCOCK and B. J. PROUT \\ Royal Cornwall Hospital (Treliske), Truro, Cornwall
}

\begin{abstract}
Simcock, A. D., and Prout, B. J. (1974). Thorax, 29, 145-146. A patient with respiratory obstruction in glandular fever. Glandular fever is a common disease, and one of the most consistent features is enlargement of tonsillar and nasopharyngeal lymph nodes. This is usually of little serious consequence and, if difficulty in swallowing or breathing occurs, responds rapidly to treatment. We report a case of glandular fever where lymph node enlargement caused respiratory obstruction which required urgent tracheostomy. Some other uncommon features are discussed.
\end{abstract}

\section{CASE REPORT}

A man aged 26 years with no significant past illnesses complained of being generally unwell with frontal headaches three weeks before admission. There was no history of contact with any infectious disease. One week before admission he was treated at home for a sore throat with penicillin and then lincomycin, with no improvement.

On admission he looked ill and had a pyrexia of $99 \cdot 4^{\circ} \mathrm{F}\left(37 \cdot 44^{\circ} \mathrm{C}\right)$. His tonsils were enlarged but there was no difficulty in swallowing, speaking or breathing. There were petechiae on the palate, and white patches were noticed on the tonsils and posterior pharyngeal wall. There was also a lymphadenopathy affecting the cervical, axillary, and inguinal lymph nodes. The liver was seen to be enlarged three fingerbreadths below the costal margin, and the spleen four fingerbreadths below the costal margin. There were no abnormal signs in the cardiovascular, respiratory or nervous systems. A clinical diagnosis of glandular fever was made.

INVESTIGATIONS The blood picture was reported as: Haemoglobin $14.8 \mathrm{~g} \%$; PCV $46 \%$; ESR $3 \mathrm{~mm} / 1 \mathrm{hr}$; WCC $13,000 / \mathrm{mm}^{3}$, of which $14 \%$ were monocytes and of which some were atypical. The Paul Bunnell test was positive. Liver function tests were normal apart from albumin $3.2 \mathrm{~g} \%$ and globulins $4.8 \mathrm{~g} \%$ (gamma globulins $2.6 \mathrm{~g} \%$ ). In the throat swab no organisms were seen and it was sterile on culture. The chest radiograph was normal and there was no sign of mediastinal lymph node enlargement.

TREATMENT AND PROGRESS The patient was treated initially with hibitane lozenges and oral prednisolone, $10 \mathrm{mg}$ six-hourly, because of the severity and extent of the lymphadenopathy, and he was sedated with diazepam, $10 \mathrm{mg}$ at night. His temperature settled in
24 hours but the tonsillar lymphoid tissues steadily enlarged ; the uvula and pharynx became increasingly swollen. Respiratory difficulties occurred four days after admission despite the prednisolone. One cyanotic attack occurred, lasting for a few minutes, and then resolved spontaneously. He was agitated and was sedated with diazepam, $10 \mathrm{mg}$ intramuscularly sixhourly. Twelve hours later he was again cyanosed with a raised respiratory rate, obstructed breathing, and a rising pulse rate.

On direct vision there was almost total obstruction of the airway at the level of the tonsils, which met in the mid-line. The uvula was also swollen, leaving only a small triangular airway between the base of the tongue and the tonsils. The patient had become dehydrated due to the development of difficulties in swallowing.

Tracheostomy through the second and third tracheal rings under local anaesthesia was undertaken on the fourth day after admission as a matter of urgency. There was an immediate improvement in the patient's colour, and normal breathing returned. Twenty-four hours later he was feeling well, but there was no reduction in the size of the swollen lymph nodes. The steroids were reduced. Four days later the lymph nodes were seen to be smaller but the left tonsil remained enlarged, red, and tense and was covered by adherent slough.

He steadily improved and without further treatment his tonsils returned to normal. Two weeks after admission he developed a typical maculopapular penicillin sensitivity rash but continued to make reasonable progress, and the steroids were further reduced. The tracheostomy was closed after 14 days. At this time the spleen and liver were becoming smaller. Three weeks after admission he was discharged feeling well. At the outpatient department four weeks later he was well and had returned to work. 


\section{COMMENT}

Complications of glandular fever were reviewed by Hobson, Lawson, and Wigfield in 1958, by Hoagland in 1960, and recently by Pullen (1973). This patient's initial symptoms before and shortly after admission were typical of the anginose type of glandular fever. Complications involving airway obstruction are rare but are reported to respond rapidly to steroids, in the doses used in this patient (Valentine, 1967; Emond, 1973). Respiratory obstruction has been reported by Hoagland (1960), recording one case, but this was considered to be due to secondary Candida infection. Otherwise respiratory obstruction in glandular fever has been attributed to oedema of the glottis or occlusion of the trachea. In the case reported, the larynx was not visible due to the almost total obstruction at tonsillar level. This had occurred despite early steroid treatment, and even though the steroids were continued for some time after tracheostomy. It is also interesting to note that the skin rash did not appear for three weeks after the initial course of penicillin given at home was begun.
It is suggested that respiratory obstruction must be borne in mind when dealing with cases of glandular fever involving gross pharyngeal lymph node enlargement. Tracheostomy may become necessary to avoid a fatal outcome in a common $\varnothing$ disease which normally carries an excellent क prognosis.

\section{REFERENCES}

Emond, R. T. D. (1973). Infectious mononucleosis. In î Medicine, Section 6, p. 452, Medical Education (International) Ltd.

Hoagland, R. J. (1960). The clinical manifestations of $\overrightarrow{0}$ infectious mononucleosis. A report of two hundred 0 cases. American Journal of the Medical Sciences, 240, 21.

Hobson, F. G., Lawson, B., and Wigfield, M. (1958). c Glandular fever: a field study. British Medical Journal, 1,845 .

Pullen, H. (1973). A new look at infectious diseases: infectious mononucleosis. British Medical Journal, 2, 350.

Valentine, W. N. (1967). Infectious mononucleosis. In Cecil-Loeb Textbook of Medicine, 12th ed., edited by P. B. Beeson and W. McDermott. Saunders, Philadelphia. 